\title{
Evaluación de materias primas utilizadas en la fabricación de baldosas de gres en el sector cerámico de Norte de Santander (Colombia)
}

\author{
Evaluation of raw materials used in the manufacture of stoneware tiles in the ceramic \\ industry from Norte de Santander (Colombia)
}

\section{Avaliação de matérias primas utilizadas na fabricação de azulejos de grés no setor cerâmico de Norte de Santander (Colômbia).}

\author{
Jorge Sánchez-Molina ${ }^{1}$, Alejandra Sarabia-Guarin² ${ }^{2}$ Diana Carolina Alvarez-Rozo ${ }^{3}$
}

Forma de citar: J. Sánchez-Molina, A. Sarabia-Guarin, D. C. Alvarez-Rozo "Evaluación de materias primas utilizadas en la fabricación de baldosas de gres en el sector cerámico de Norte-de-Santander (Colombia)", Respuestas, vol. 21, no. 2, pp. 48-56, 2016.

Recibido:

Febrero 18 de 2016

Aceptado:

Mayo 27 de 2016

\section{Resumen}

Antecedentes: Los municipios de Cúcuta, Los Patios, Villa del Rosario, El Zulia y San Cayetano, representan el 10\% de la extensión del departamento de Norte de Santander, y el $6,85 \%$ de su territorio lo ocupa la industria cerámica, la cual produce todo tipo de productos derivados de la arcilla; mineral cuyas características físicas, químicas y mineralógicas varían ampliamente, por lo que el control de la calidad de los productos empieza por la caracterización y control de calidad de sus materias primas. Objetivo: Caracterizar mediante análisis químicos, mineralógicos y físico-cerámicos tres materias primas utilizadas en la fabricación de productos de gres: arcilla, chamota y caliza provenientes del departamento Norte de Santander. Métodos: Se caracterizaron las materias primas mediante las técnicas de DRX, FTIR, y ensayos físico-cerámicos que incluyen contracción en secado, contracción en cocido, absorción de agua, pérdidas por calcinación y porcentaje de arena. Posteriormente por extrusión y prensado se obtuvieron probetas de tres tipos de mezclas referenciadas como M1, M2 y M3, con diferente composición de material arcilloso, chamota y caliza, luego se sinterizaron a $1050^{\circ} \mathrm{C}-1250^{\circ} \mathrm{C}$ y se analizó la absorción de agua y el porcentaje de contracción en la curva de gresificación. Resultados: El patrón de DRX muestra la presencia de cuarzo y caolinita en la arcilla, y de calcita y cuarzo en la caliza, lo cual se evidencia en el espectro FTIR. El comportamiento físico-cerámico de las mezclas realizadas evidenció valores significativamente bajos de absorción de agua (inferior al 6\%) con temperaturas de cocción de entre $1150^{\circ} \mathrm{C}$ y $1250^{\circ} \mathrm{C}$. Conclusión: Se identificó que las materias primas evaluadas son aptas para la fabricación de baldosas de gres, siendo M3-E la formulación más óptima, compuesta por $80 \%$ material arcilloso, 15\% chamota y 5\% caliza (pasante malla 100), y moldeada por extrusión.

Palabras clave: arcilla, absorción de agua, caliza, difracción de rayos x, espectroscopia infrarroja.
Abstract
Background: The municipalities of Cucuta, Los Patios, Villa del Rosario, Zulia and San Cayetano, representing 10\% of the department's extension of Norte de Santander, and $6.85 \%$ of its territory is occupied by the ceramic industry, which produces all kinds of products derived from clay; mineral whose physical, chemical and mineralogical characteristics vary widely, so the quality control of products, beginning with the characterization and 
quality control of raw materials. Objective: To characterize by chemical, mineralogical, and physico-ceramic analysis three raw materials used in the manufacture of ceramic: clay, fire clay and limestone from the Norte de Santander department. Methods: raw materials were characterized by techniques of DRX, FTIR, and physical-ceramic tests including shrinkage in drying shrinkage cooked, water absorption, loss on ignition and percentage of sand. Subsequently they extruded and pressed samples of three types of mixtures referenced as M1, M2 and M3, with different composition of clay, fire clay, limestone, then were sintered at $1050^{\circ} \mathrm{C}-1250^{\circ} \mathrm{C}$ and the behavior of water absorption and the percentage shrinkage was analyzed in vitrification curve. Results: The pattern of DRX shows the presence of quartz and kaolinite in the clay and calcite and quartz in the limestone, which is evident in the FTIR spectrum. The physical-ceramic behavior of mixtures made significantly low showed water absorption values (less than 6\%) with firing temperatures between $1150^{\circ} \mathrm{C}$ and $1250^{\circ} \mathrm{C}$. Conclusion: Was identified that assessed raw materials are suitable for manufacturing of stoneware tiles, being M3-E the most optimal formulation composed of $80 \%$ clay material, $15 \%$ grog, and 5\% limestone (through 100 mesh), and molded by extrusion.

Keywords: clay, calcite, x-ray diffraction, infrared spectroscopy, water absorption

\section{Resumo}

Antecedentes: Os municípios de Cúcuta, Los Patios, Villa del Rosario, El Zulia e San Cayetano, representam o 10\% da extensão do departamento de Norte de Santander, e o 6,85\% de seu território é ocupado pela indústria cerâmica, a qual produz todo tipo de produtos derivados da argila; mineral cujas características físicas, químicas e mineralógicas variam amplamente, pelo que o controle da qualidade dos produtos começa pela caracterização e controle de qualidade de suas matérias primas. Objetivo: Caracterizar através da análise química, mineralógica e físico-cerâmica três matérias primas utilizadas na fabricação de produtos de grés: argila, chamote e calcário originário do Estado Norte de Santander. Métodos: Caracterizaram-se as matérias primas através das técnicas: difração de raios- $\mathrm{X}$ (DRX), infravermelho com transformada de Fourier (FTIR), e ensaios físico-cerâmicos que incluem contração em secagem, contração em cozido, absorção de agua, perdidas por calcinação e percentagem de areia. Posteriormente, por extrusão e prensado obtiveram-se provetas de três tipos de misturas referenciadas como $\mathrm{M}_{1}, \mathrm{M}_{2} \mathrm{e} \mathrm{M}_{3}$, com diferente composição de material argiloso, chamote e calcário. Logo após, se sintetizaram a $1.050^{\circ} \mathrm{C}-1.250^{\circ} \mathrm{C}$, sendo analisada a absorção de agua e a percentagem de contração na curva de gresificação. Resultados: O padrão de DRX mostrou a presença de quartzo e caulinita na argila, e de calcite e quartzo no calcário, o qual se evidenciou no espectro FTIR. O comportamento físico-cerâmico das misturas realizadas evidenciou valores significativamente baixos de absorção de agua (inferior ao 6\%) com temperaturas de coç̧ão de entre $1.150^{\circ} \mathrm{C}$ e $1.250^{\circ} \mathrm{C}$. Conclusão: Identificou-se que as matérias primas avaliadas são aptas para a fabricação de azulejos de grés, sendo $\mathrm{M}_{3}$-E a melhor formulação, composta por $80 \%$ de material argiloso, $15 \%$ chamote e $5 \%$ calcário (malha 100 ), moldada por extrusão.

Palavras-chave: absorção de agua, argila, calcário, difração de raios-X, infravermelho com transformada de Fourier

\section{Introducción}

El Departamento Norte de Santander se caracteriza por tener abundantes yacimientos de arcilla, la cual es empleada como materia prima en el proceso de fabricación de diversos productos cerámicos como ladrillo, bloque, teja, entre otros [1]. Estas arcillas presentan propiedades físicas y químicas apropiadas para su utilización en la industria cerámica 
No. 2

Jul - Dic 2016 ISSN 0122-820X E-ISSN 2422-5053

PP: 48-56
[2]. Es de resaltar que las propiedades de las materias primas empleadas en la fabricación de productos cerámicos, pueden afectar directamente los parámetros del proceso y las características del producto final [3]. Por tal motivo, es de gran importancia caracterizar las materias primas con el fin de mejorar la eficacia y eficiencia del proceso y obtener productos que cumplan con las propiedades exigidas para su comercialización.

Es importante destacar que los productos de gres son compactos, poco absorbentes, refractarios, aislantes eléctricos y su porosidad es bastante baja (inferior al $4 \%$ ), se fabrican con arcillas de alto potencial de vitrificación a bajas temperaturas. Su porosidad y su impermeabilidad se deben a la combinación entre sílice libre, aluminio álcalis y silicatos de aluminio, sustancias que se solidifican después de la fusión [4].

Partiendo de estas observaciones, la presente investigación se dirigió hacia el análisis de los resultados de la caracterización físicoquímica de tres materias primas: arcilla, caliza y chamota, provenientes del departamento de Norte de Santander, Colombia. Considerando su potencial aplicación, en la fabricación de baldosas de gres, que tienen estas materias primas por su calidad y potencial uso en la fabricación de materiales de construcción [5], la pesquisa se complementó con la realización de ensayos físico-cerámicos (contracción en secado, contracción en cocido, absorción de agua, pérdidas por calcinación y porcentaje de arena).

\section{Materiales y métodos}

\subsection{Materias primas}

Para efectos de la investigación se seleccionaron las materias primas empleadas en la fabricación de baldosas cerámicas, estas son: arcilla, caliza y chamota. Se recolectaron aproximadamente $100 \mathrm{Kg}$ de arcilla de la mina de arcilla La Alejandra, la cual hace parte de la formación Guayabo, y está localizada en la vereda La Alejandra, municipio del Zulia, Norte de Santander; la chamota cocida de bloque empleada como desgrasante, proviene de una empresa ubicada en la vereda La Alejandra, municipio del Zulia, Norte de Santander y, la caliza es proveniente del municipio de Los Patios.

A las muestras de arcilla obtenidas se le aplicó un proceso de trituración primaria para obtener un tamaño de partícula entre $20-30 \mathrm{~mm}$, luego se le realizó molienda seca por medio de un molino de martillos para conseguir un tamaño de partícula de $0,5 \mathrm{~mm}$

\subsection{Caracterización mineralógica de materias primas mediante DRX y FTIR}

Las muestras de arcilla y caliza fueron caracterizadas por Difracción de Rayos $\mathrm{X}$ utilizando el método de polvo en un difractómetro (marca BRUKER modelo D8 Advance con geometría DaVinci). El análisis cualitativo de las fases presentes en la muestra se realizó mediante comparación del perfil observado con los perfiles de difracción reportados en la base de datos PDF-2 del Internacional Center for Diffraction Data (ICDD). El análisis de las fases encontradas se realizó mediante el refinamiento por el método de Rietveld.

En la espectroscopía infrarroja por transformada de Fourier se empleó un equipo Thermo Nicolet ${ }^{\mathrm{TM}}$, modelo FTIR iS ${ }^{\mathrm{TM}} 10$, y en el conformado de las pastillas de $\mathrm{KBr}$ se utilizó una prensa manual marca Nicolet $^{\mathrm{TM}}$. Las condiciones de trabajo fueron: infrarrojo de 400 a $4000 \mathrm{~cm}^{-1}$, lectura en $\mathrm{X}$ long onda, resolución $0,4 \mathrm{~cm}^{-1}$ y número de escaneos igual a 32. El software utilizado fue el OMNIC.

\subsection{Caracterización de prototipos}

Se formularon tres tipos de mezclas referenciadas como: M1, M2 y M3, a las cuales se les determinó contracción en cocido 
y absorción de agua, a temperaturas de 1050$1100-1150-1200$ y $1250^{\circ} \mathrm{C}$. La muestra M1 se elaboró como elemento patrón de comparación, por tanto fue preparada solo con material arcilloso (pasante malla 12), la muestra M2 corresponde $85 \%$ material arcilloso y $15 \%$ chamota (pasante malla 12 ) y la muestra M3 corresponde $80 \%$ material arcilloso, $15 \%$ chamota y $5 \%$ caliza (pasante malla 100).

Las probetas se obtuvieron bajo dos técnicas diferentes de conformado: extrusión y prensado, para lo cual se usó la extrusora modelo New Wave serie 101 y la prensa hidráulica modelo GKM-1, marca Gabrielly Technology, respectivamente. Una vez extruidas y prensadas las probetas se dejaron secar al aire libre durante dos días, posteriormente se pasaron a la estufa de secado a una temperatura de $110^{\circ} \mathrm{Chasta}$ obtener un peso constante, con el fin de evaporar el agua presente en la mezcla. Teniendo en cuenta que la arcilla se contrae durante el proceso de secado, se midieron nuevamente las dimensiones de las probetas con el propósito de determinar el porcentaje de contracción en secado.

Los análisis físico-cerámicos fueron desarrollados a escala laboratorio en el Centro de Investigación de Materiales Cerámicos (CIMAC) de la Universidad Francisco de Paula Santander (UFPS), teniendo en cuenta el proceso productivo de las empresas fabricantes de productos cerámicos del área metropolitana de Cúcuta.

\section{Resultados y análisis}

\subsection{Caracterización mineralógica mediante DRX y FTIR}

El patrón de difracción de rayos $\mathrm{X}$ de la muestra arcillosa proveniente de la mina La Alejandra muestra un contenido de $33,6 \%$ en cuarzo (Figura 1); este mineral no arcilloso, se encuentra naturalmente $y$ en mayor cantidad que los demás minerales presentes [6]. El cuarzo contribuye a disminuir la plasticidad de la mezcla de materia prima, aumentar la permeabilidad de la pieza cruda y el coeficiente de dilatación de la pieza cocida. $\mathrm{Su}$ alto contenido de cuarzo lo convierte en un material arenoso, que puede utilizarse como material desgrasante para la preparación de las pastas [7].

En la Figura 1 se observa la presencia de caolinita $(19,3 \%$ en peso) de forma mas representativa en el plano 001, lo que coincide con lo expuesto por SACMI [8] quienes reportan que se forman picos de este mineral en las siguientes posiciones angulares: $12,5^{\circ}(001) ; 24,9^{\circ}(002) ; 37,7^{\circ}$ $(003) ; 51,1^{\circ}(004)$. La caolinita es el principal mineral de arcilla utilizado en la fabricación de productos cerámicos, debido a que este influye en el proceso cerámico favoreciendo la homogeneización de la humedad, baja contracción y absorción de agua, baja moldeabilidad, secado fácil y cocción a altas temperaturas [9]

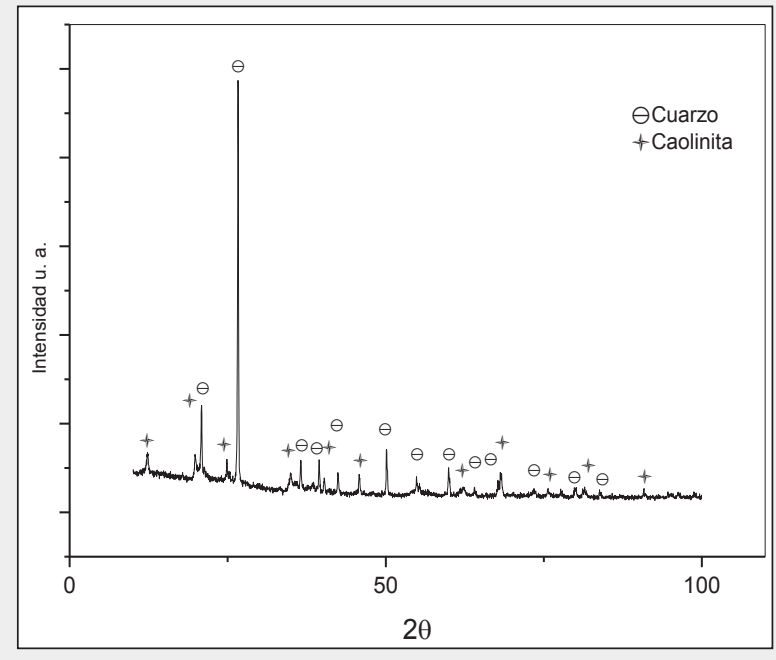

Figura 1. Análisis mineralógico de la arcilla La Alejandra.

$$
\text { Fuente: Autor }
$$

El patrón de Difracción de Rayos X de la muestra de la caliza (Ver Figura 2) muestra la presencia de calcita en $75,1 \%$ y de cuarzo con $3,2 \%$. Un incremento en el porcentaje de carbonato calcico de la pasta trae consigo un aumento en la absorción de agua y un 
No. 2

Jul - Dic 2016 ISSN $0122-820 \mathrm{X}$

E-ISSN 2422-5053

PP: $48-56$ retraso en el inicio de la sinterización por formación de fase líquida [6]. Esto se debe a que a temperaturas del orden de los 800$900^{\circ} \mathrm{C}$, se produce la descomposición de la calcita en óxido calcico con la silice y la alúmina, procedentes de la deshidroxilación de los minerales arcillosos, y con el cuarzo y feldespatos de pequeño tamaño de partícula para formar fases cristalinas cálcicas (silicatos y silicoaluminatos de calcio). Por lo tanto, si bien la utilización de carbonatos o arcillas calcareas en la fabricación de revestimientos porosos esta justificada por la doble función que las fases cálcicas desempeñan: regulan la porosidad, proporcionando un intervalo de cocción amplio a estas pastas y reducen la expansión por humedad.

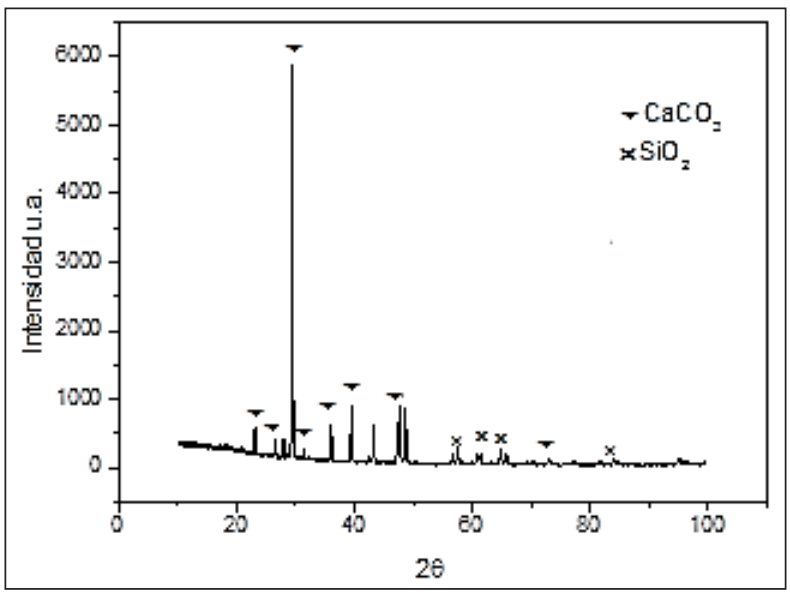

Figura 2. Análisis mineralógico de la caliza. Fuente: Autor

Los espectros adquiridos de la caracterización por FTIR en las muestras de Arcilla y Caliza son presentados en la Figura 3.

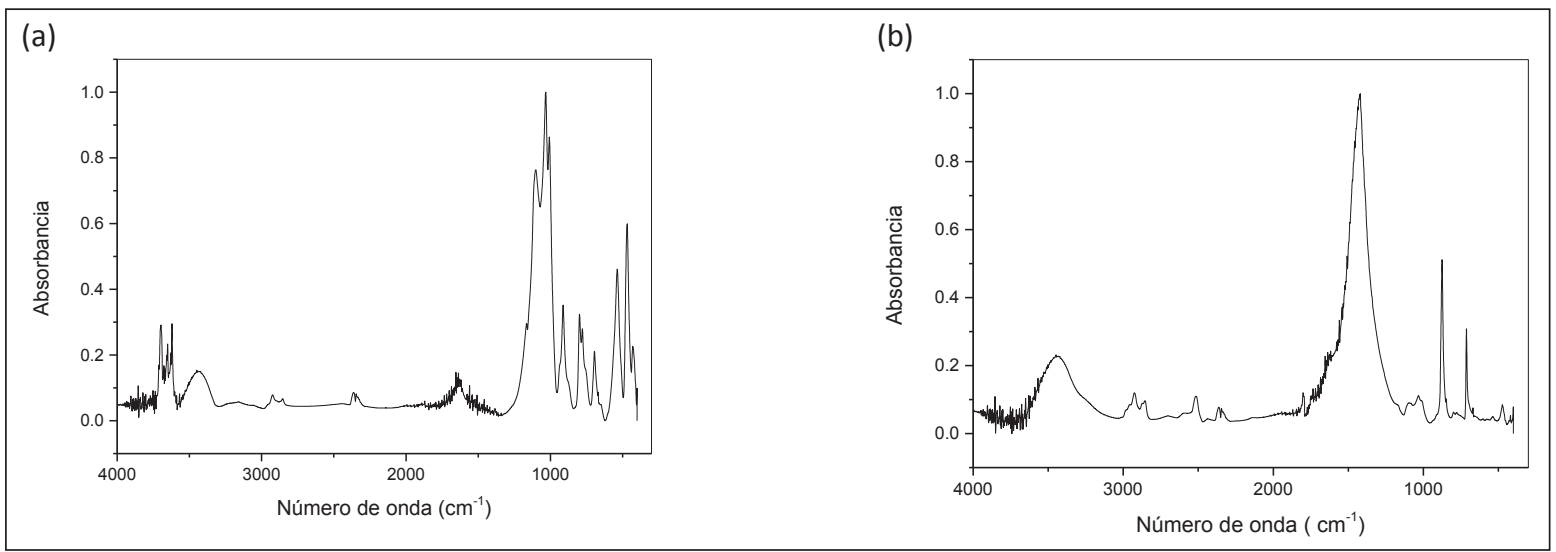

Figura 3. (a) Espectro FTIR de la arcilla Alejandra. (b) Espectro FTIR de la caliza. Fuente: Autor

Nótese que en el espectro de FTIRse tuvieron en cuenta las bandas observadas en el rango de 670 a $4000 \mathrm{~cm}^{-1}$, es decir en una frecuencia de infrarrojo medio, identificándose para la arcilla La Alejandra, varias formas de minerales presentes [10]. Las vibraciones de estiramiento de la fase cuarzo fueron pre- sentadas a $1090 \mathrm{~cm}^{-1}, 798.60 \mathrm{~cm}^{1}, 695.14 \mathrm{~cm}^{1}$ y $468.21 \mathrm{~cm}^{1}$, la fase illita $/$ moscovita fueron presentadas a $3620.30 \mathrm{~cm}^{-1}, 3447.88 \mathrm{~cm}^{-1}$ y $537.21 \mathrm{~cm}^{-1}$; y por último la fase caolinita fue presentada a $3697.04 \mathrm{~cm}^{-1}$. En la Figura 3(b) se evidencia una fase pronunciada de la calcita correspondiente a bandas ubica- 
das a $1426 \mathrm{~cm}^{-1}, 2925.15 \mathrm{~cm}^{-1}, 875.28 \mathrm{~cm}^{-1} \mathrm{y}$ $712.58 \mathrm{~cm}^{-1}$.

\subsection{Caracterización físico cerámica de las materias primas}

\subsubsection{Contenido de humedad}

El contenido de humedad determina el porcentaje de humedad (agua) que contiene una materia prima [11]; esta se expresa en base húmeda o en base seca [12]. En la Tabla I se reportan los diferentes porcentajes de humedad de la arcilla, caliza y chamota usadas como materias primas en el presente estudio.

Tabla I. Contenidos de humedad para las materias primas seleccionadas

\begin{tabular}{cc}
\hline Muestra & Contenido de humedad (\%) \\
\hline Arcilla La Alejandra & 3,4 \\
Caliza & 4,5 \\
Chamota & 2,8 \\
\hline & Fuente: Autor
\end{tabular}

En la Tabla I se evidencia que los materiales presentaron una humedad relativamente baja en referencia a la humedad ideal $(10 \%)$ que deben contener las materias primas para llevar a cabo los ensayos [12] posteriormente se seco a peso constante los materiales en la estufa de secado durante 24 horas, para garantizar la eficiencia de la molienda.

\subsubsection{Retenido sobre tamiz}

El retenido sobre tamiz se estimó aplicando la norma ASTM C325-81. La aplicación de la norma permite establecer para la muestra de arcilla, valores de tamiz malla 230 (abertura de $63 \mu \mathrm{m})$, obteniéndose como resultado que la arcilla contiene un 7,00\% de arena, describiéndolo como un material altamente plástico, lo cual puede ocasionar problemas en la etapa de secado. Para el presente estudio es un buen parámetro porque la caliza empleada para las diversas muestras no es plástica o desgrasante.

\subsubsection{Pérdidas por calcinación de la caliza}

Con las pérdidas por calcinación se determinó la cantidad de carbonato de calcio $\left(\mathrm{CaCO}_{3}\right)$ que compone la caliza, lo que permite anticipar su comportamiento durante la cocción de los productos cerámicos a temperatura final de $950^{\circ} \mathrm{C}$ durante dos horas. En la Tabla II se reportan los valores por pérdidas de calcinación para la caliza, con base a los cuales, se puede deducir que es un material técnicamente puro.

Tabla II. Valores de pérdidas de calcinación para el material caliza

\begin{tabular}{|c|c|c|c|c|c|}
\hline $\begin{array}{l}\text { Masa del crisol } \\
\text { (m1) }\end{array}$ & $\begin{array}{c}\text { Masa de la } \\
\text { muestra }(\mathbf{m} 2)\end{array}$ & $\begin{array}{c}\text { Masa m1 } \\
\text { m2 }\end{array}$ & Masa de calcinación & $\begin{array}{l}\text { Masa de la muestra } \\
\text { después de la calcinación }\end{array}$ & Pérdidas por calcinación \\
\hline G & G & g & g & G & $\%$ \\
\hline 23,5797 & 2,0000 & 25,5797 & 24,7553 & 1,1756 & 41,22 \\
\hline
\end{tabular}

Un material calizo se considera puro cuando el contenido de $\mathrm{CaCO}_{3}$ está entre 39 y $42 \%$ de pérdidas de calcinación [13]. Al ser mezclado en una formulación, permite mejorar las propiedades de adherencia, caso de las baldosas, e incluso puede controlar el color de quema y las dimensiones del producto terminado.

\subsection{Caracterización de prototipos}

\subsubsection{Curvas de gresificación}

En la Figura 4, se aprecia que los prototipos obtenidos por extrusión presentan mayores valores de contracción en comparación a los obtenidos por prensado; obteniéndose 
No. 2

Jul - Dic 2016 ISSN 0122-820X

E-ISSN 2422-5053

PP: 48-56 los valores más bajos para $\mathrm{M} 3$, tanto en extruido (M3-E) como en prensado (M3-P), las cuales contienen $5 \%$ de caliza, que le proporciona a la mezcla estabilidad en las dimensiones de la pieza durante la cocción [6]. Se observa además que las mezclas tienen su punto de gresificación aproximadamente a temperaturas alrededor de $1100^{\circ} \mathrm{C}$ y $1150^{\circ} \mathrm{C}$.

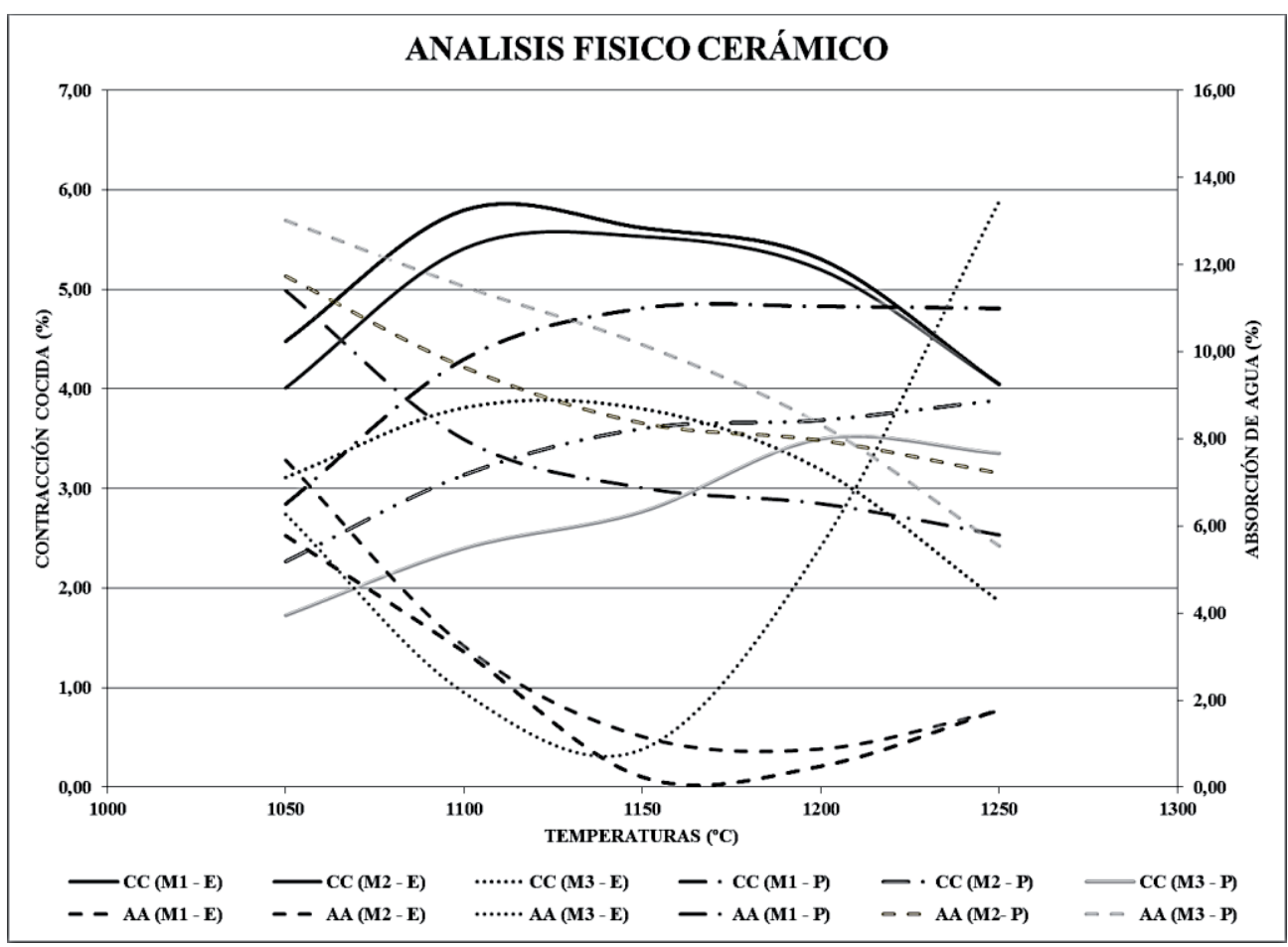

Figura 4. Curvas de gresificación de M1-M2 y M3 (extrusión y prensado)

Fuente: Autor

\subsubsection{Absorción de agua}

En la Figura 5 se observa que la M1-E presentó valores muy bajos de absorción de agua, inferiores $3 \%$, para temperaturas de $1100^{\circ} \mathrm{C}$ y $1250^{\circ} \mathrm{C}$, lo cual la ubica en el grupo AIa según la ISO 13006 [14]. La mezcla M3-E presenta un incremento de la absorción 54 de agua debido a la presencia de caliza en su composición, obteniéndose sus valores más bajos a $1150^{\circ}$ y $1200^{\circ} \mathrm{C}$. La caliza regula la porosidad, proporcionando un intervalo de cocción amplio a las pastas empleadas en la fabricación de productos gresificados, disminuyendo el intervalo de vitrificación, además de favorecer la deformación de las piezas durante la cocción [6].
La causa de que los valores de absorción de agua de las probetas prensadas sean más altos que los de las probetas extruidas en todas las temperaturas, puede encontrarse en el grado de compactación de las probetas, debido a la granulometría de la arcilla [15], a una menor presión de prensado y a la posición de las mismas dentro del horno; este último criterio puede verse reflejado además en el porcentaje de contracción, el cual será inferior en referencia a las probetas extruidas, a menor contracción, mayor absorción de agua. Los altos porcentajes de absorción de agua, indican mayor porosidad, lo que facilita la evaporación y salida del agua durante la etapa de cocción. 


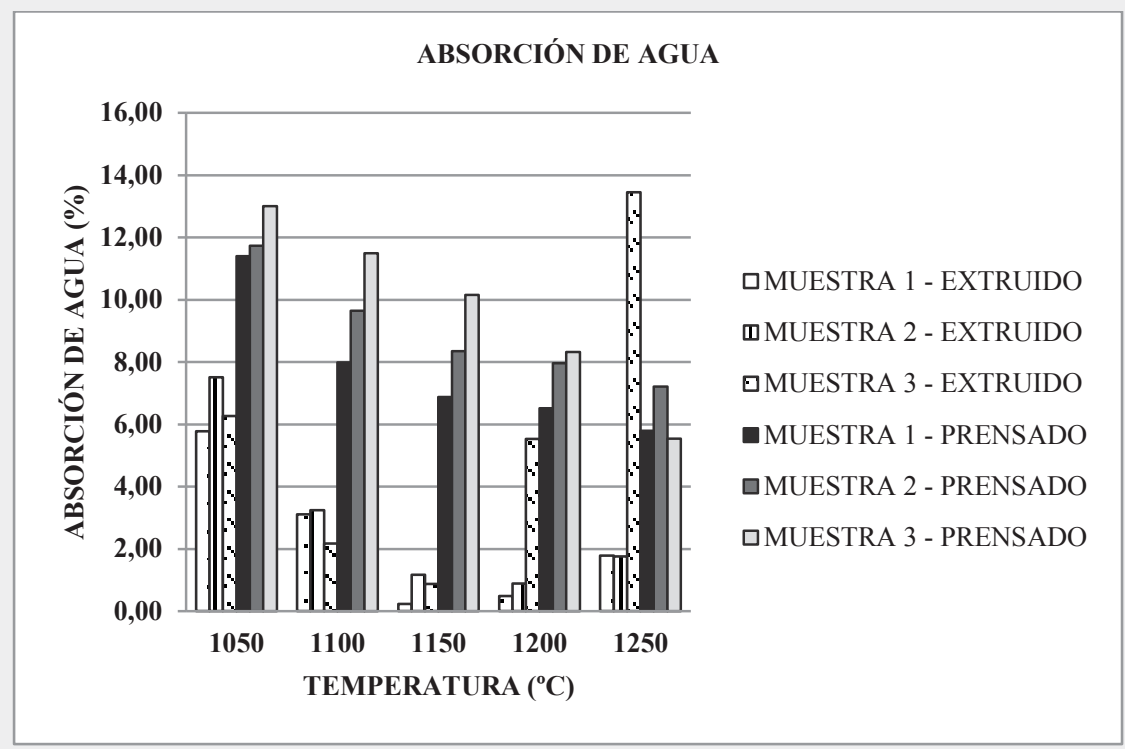

Figura 5. Comparativo de las muestras Fuente: Autor

\section{Conclusiones}

Con respecto a la arcilla de la mina La Alejandra se evidenció el predominio de las fases cuarzo y caolinita; donde los valores de absorción de agua de M1 son inferior al 5\% en las temperaturas entre 1150 y $1200^{\circ} \mathrm{C}$.

La porosidad del material disminuye en las formulaciones con un contenido de caliza menor al $5 \%$, a temperatura de $1100^{\circ} \mathrm{C}$ la absorción disminuye mientras la contracción aumenta, el punto de gresificación se encuentra a temperatura de $1200^{\circ} \mathrm{C}$ por lo cual a $1250^{\circ} \mathrm{C}$ se evidencia un considerable aumento en la absorción de agua de 13,45\% y una disminución en la contracción en cocido de $1,87 \%$.

$\mathrm{Al}$ analizar el comportamiento de las materias primas en el proceso de cocción se dedujo, que el punto de gresificación de M1 está muy cercano tanto en el proceso de extrusión como en el de prensado $\left(1050^{\circ} \mathrm{C}-1100^{\circ} \mathrm{C}\right)$, a diferencia de $\mathrm{M} 3$ en donde el punto de gresificación se encuentra a las temperaturas de $1200^{\circ} \mathrm{C}$.

La muestra M2 por extrusión presenta el valor más bajos de absorción de agua $(1,76 \%)$ a la temperatura de $1250^{\circ} \mathrm{C}$, lo cual indica que tiene un alto contenido de partículas finas que vitrifican con mayor facilidad en este tipo de conformado, es decir esta mezcla es la ideal para la fabricación de baldosas de gres vitrificadas.

El diagrama de contracción lineal y absorción de agua, permitió estudiar el comportamiento de las mezclas durante la cocción, siendo de gran ayuda para identificar que la formulación más óptima para la fabricación de baldosas en gres es M3-E, compuesta por 80\% material arcilloso, $15 \%$ chamota y $5 \%$ caliza (pasante malla 100), y moldeada por extruido,

\section{Referencias}

[1] J. Sánchez. "Caracterización físico química de las arcillas rojas del área metropolitana de Cúcuta". Proyecto para el ascenso en el escalafón de la categoría Asociado a Titular. Departamento de Química, Facultad de ciencias básicas, Universidad Francisco de Paula Santander, 2014.

[2] Grupo de Investigación en Tecnología Cerámica GITEC. "Conformación e implementación del clúster de la 
No. 2 cerámica en Norte de Santander". Universidad Francisco de Paula Santander. Facultad de ciencias Básicas. Anexo A. Pág. 5. 2008.

[3] T. Poirier, J. Baena, C. Picardo and X. Hung. "Relevancia del factor "materia prima" en un proceso cerámico industrial: Un caso de estudio venezolano", Revista Facultad de Ingeniería Universidad Central de Venezuela, vol.29, no.3, pp. 103-113, 2014.

[4] Asociación nacional de fabricantes de ladrillo y derivados de la arcilla ANFALIT. "Manual técnico para construcción de muros divisorios y acabados arquitectónicos con productos de arcilla”. Bogotá: Instituto Colombiano de la Arcilla, 2007.

[5] Red Clúster Colombia. "Arcillas Competitivas: Entregable 2", Mayo 2013. [En Línea]. Disponible en: http:// redclustercolombia.com/clusters-encolombia/iniciativa/10

[6] A. Barba, V. Beltrán, C. Felíu, et al. "Materias primas para la fabricación de soportes de baldosas cerámicas". Castellón, España: Instituto de Tecnología Cerámica ITC, 2002.

[7] J. A. Santos, P. Y. Malagon and E. M. Cordoba. "Caracterización de arcillas y preparación de pastas cerámicas para la fabricación de tejas y ladrillos en la región de Barichara, Santander". Dyna, vol. 78, no. 167, 2011, pp. 53-61, 2009.

[8] SACMI. "Tecnología de la fabricación de azulejos". Asociación técnicos cerámicos del centro experimental de Sacmi Imola. II-25. Castellón, España: Generalitat Valenciana IMPIVA. 1990.

[9] H. Mesbah, M. A. Wilson, M. A. Carter and J. Shackleton. "Effect of prolonged sintering time at $1200^{\circ} \mathrm{C}$ on the phase transformation and reactivity with moisture of fired kaolinite". En 11th International Conference on Ceramic Processing Science, ICCPS-11, (Zurich, Switzerland), The University of Manchester Library, 2010.

[10] L. Vaculíková and E. Plevová. "Idetification of clay minerals and micas in sedimentary rocks". Acta Geodyn. Geomater, vol 2, no. 2, pp.167-175. 2005.

[11] ASTM C 324-2001. Standard Test Method for free moisture in ceramic whiteware clays. 2014.

[12] J. Amorós, E. Sánchez, J. García-Ten, et.al. "Manual para el control de la calidad de materias primas arcillosas". Castellón, España: Instituto de Tecnología Cerámica ITC. 1998.

[13] D. Monsalve and P. Ospina. "Implementación de los análisis químicos y elaboración del manual de laboratorio para las materias primas utilizadas en la preparación de pastas cerámicas en la empresa Cerámica Italia S.A." Trabajo de grado Tecnología Química, Universidad Francisco de Paula Santander. Cúcuta, Colombia. 2001.

[14] Ceramic tiles: Definitions, classification, characteristics and marking. ISO 13006, 2012.

[15] J. M. Villora, P. Callejas and M. F. Barba. "Influencia del procesamiento de materiales cerámicos en su comportamiento como adsorbente de iones en aguas industriales". Boletín de la Sociedad Española de Cerámica y Vidrio, vol. 43, no.1, pp. 75-79, 2004. 